\title{
Reply to Beall and Priest
}

\author{
Matti Eklund \\ Departanent of Philosophy \\ Cornell University \\ me72@cornell.edu \\ Received by Greg Restall \\ Published November 7, 2008 \\ http://www.philosophy . unimelb.edu.au/ajl/2008 \\ (C) 2008 Matti Eklund

\begin{abstract}
In my "Deep Inconsistency" (Australasian Journal of Philosophy, 2002), I compared my meaning-inconsistency view on the liar with Graham Priest's dialetheist view, using my view to help cast doubt on Priest's arguments for his view. Jc Beall and Priest have recently published a reply to my article (Australasian Journal of Logic, 2007). I here respond to their criticisms. In addition, I compare the meaning-inconsistency view with Anil Gupta and Nuel Belnap's revision theory of truth, and discuss how best to deal with the strengthened liar.
\end{abstract}

\section{INTRODUCTION}

In my 'Deep Inconsistency' (2002a) (henceforth DI), I criticized Graham Priest's dialetheism by unfavorably comparing it to my preferred view on the liar paradox, a view I will here call the meaning-inconsistency view. Perhaps

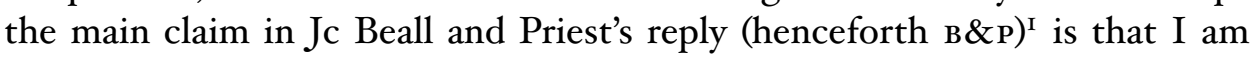
guilty of an ignoratio: in DI, I argue that Priest (1987) fails to establish the analyticity of certain principles, but, B\&P say, Priest (I987) isn't concerned to argue for the analyticity of these principles. Among other criticisms B\&P level against DI can be mentioned especially the following: (i) Since I do not in fact defend a particular theory of truth I am 'out of the game', not really participating in the debate which others participate in; (ii) I lack-for principled reasons-an account of in virtue of what principles are meaning-constitutive.

Here is what I will do in this reply. First I will briefly rehearse the main elements of both my own view and the criticisms of dialetheism raised in DI. Then I will respond to the charges listed from в\&P. Lastly I will make some remarks on the strengthened liar.

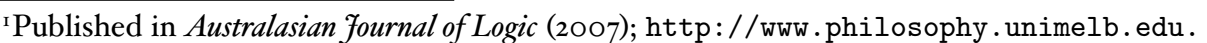
$\mathrm{au} / \mathrm{ajl} / 2007 /$ 


\section{THE MEANING-INCONSISTENCY VIEW}

Let a meaning-constitutive principle (henceforth, an $m c$ ) be-roughly; some needed clarification will be provided below - a principle such that it is part of competence with the expressions involved to be disposed to accept it. On my view, some mcs are not true, hence not analytically true. Specifically, this is shown by some paradoxes. Both the liar and the sorites paradoxes arise because there are mcs which jointly lead to contradiction, and hence cannot all be true. As I put it in DI, these paradoxes show our language to be deeply inconsistent. (Another label, perhaps a better one, suggested by Brian Weatherson in his weblog, may be 'meaning-inconsistency'.) Somewhat simplifiedly and somewhat metaphorically we may put the matter as follows. To employ an expression with a certain meaning is to, so to speak, take on certain semantic obligations: to use the expression in accordance with the rules for its use, the mcs. But what can happen is that the obligations taken on are inconsistent: there is nothing a speaker can do to fulfill all her obligations. Compare, perhaps, someone who has taken on inconsistent moral obligations-having made promises she cannot possibly jointly keep. (The talk of 'rules' must be taken with a grain of salt. In my view, talk of mcs should replace talk of rules.) On views of meaning which accord pride of place to something analogous to mcsthis is, more or less, all views according to which meaning is conceptual-rolethere is a separate question of what the truth conditions of sentences are and what the semantic values of subsentential expressions-their contributions to truth conditions-are. The standard answer is that the semantic values are such as to make true the principles that are part of conceptual role. If, like me, one allows that mcs may fail to be true, one cannot give this standard answer unmodified. What I say instead is: the semantic values are such as to make the mcs, taken jointly, as close to true as possible (given other constraints) 3

The idea is helpfully explained by considering the traditional notion of analyticity. As theorists like Paul Boghossian (e.g. 1996) and Jamie Tappenden (e.g. 1993) have stressed, there are several distinct ideas underlying the traditional notion of analyticity. One is the metaphysical idea of truth in virtue of meaning. The other concerns what it is part of semantic competence to be disposed to accept. Once these ideas are properly distinguished, the suggestion that some sentences can have the second property without having the firstand without even being true-is fairly natural. It is the latter property I call meaning-constitutivity (with the proviso that more care is required in a fully

\footnotetext{
${ }^{2}$ And in my (2007) there is a more comprehensive discussion of the issue.

${ }^{3}$ B \& P complain (p. $75 \mathrm{fn} 2$ ) about the label 'deep inconsistency', saying that the inconsistency in question 'is of only a relatively superficial kind'. There isn't much point to quarreling over labels. But just to explain the use of the label: the reason the inconsistency is deep is that on this view, when the language is inconsistent, there is nothing a speaker can do to satisfy all her semantic obligations. By contrast, on the inconsistency view of a typical dialetheist, a speaker can satisfy all her semantic obligations: it is just the obligations are different from what they are often taken to be.
} 
accurate statement); I will reserve the label analyticity for the former property. Analyticity then implies truth, while it is a central claim of the meaninginconsistency view that meaning-constitutivity doesn't.

\section{COMPARISON WITH THE REVISION THEORY}

To further explain what the meaning-inconsistency view does and does not involve, it may help to compare it with Gupta and Belnap's (I993) revision theory of truth.

On Gupta and Belnap's theory, a sentence $S$ is definitionally equivalent to 'S is true', but the two are not always materially equivalent. It may then sound as if Gupta and Belnap defend a theory not too different from the one I defend. That is, it may sound as if their claim of definitional equivalence amounts to saying that it is meaning-constitutive for 'true' that ' $\mathrm{s}$ ' is true' and 's' are materially equivalent, even when ' $s$ ' is true' and 's' are not actually materially equivalent. However, there are fundamental differences between Gupta and Belnap's revision theory and the meaning-inconsistency view, and it may be instructive to see what they are.

Consider an example Gupta and Belnap use to illustrate their theory: the predicate $\mathrm{G}$, introduced by the following definition.

(I) $x$ is $G=_{\text {def }} x$ is $F$ or $x$ is both $H$ and non-G.

We are attempting to define the concept $G$, and $G$ itself occurs in the definiens so this is a circular definition. But the definition is non-trivial. In fact, so long as there are no non-Fs which are $\mathrm{H}$, nothing untoward happens at all. If $a$ is an $F$, then it follows immediately from the definition that it is $G$. If $\mathrm{a}$ is a non- $\mathrm{F}$ and a non- $\mathrm{H}$ then it follows that a is non-G. But suppose that $\mathrm{a}$ is non- $F$ and $H$. Then it would appear that by familiar reasoning we can derive a contradiction. Suppose a is G. We can infer the definiens from the definiendum, and since $a$ is by assumption $G$ and a fails to be $F$, $a$ is $H$ and non-G. So a is non-G, which contradicts the assumption we began with. So a is non-G. But then $a$ is $H$ and non- $G$ and by the definition, $a$ is $G$. So a is both $G$ and non-G. Contradiction!

But Gupta and Belnap hold that while ordinarily, definitions like (I) are held to amount to

(2) $x$ is $G$ if and only if $x$ is $F$ or $x$ is both $H$ and non-G,

we should not conceive of definitional identities like (I) as biconditionals but instead as rules licensing the move from the left hand side to the right hand side and vice versa.

The idea is this. Suppose that we come to (I) with the hypothesis that nothing is $\mathrm{G}$. Then a will by assumption be both $\mathrm{H}$ and non-G. Then (I) licenses us instead to conclude that a is $\mathrm{G}$. This is not to be conceived of as a 
contradiction but as a revision. And if we come to (I) with the hypothesis that $a$ is $G$, then since $a$ is not $F, a$ is $H$ and non-G; so a non-G after all.

Similarly for the liar. Gupta and Belnap say that when we reason our way to a contradiction, that is because we have mistakenly taken definitional equivalence to entail material equivalence.

Now, the difference between the revision theory as Gupta and Belnap defend it and the meaning-inconsistency view is the following. There is nothing in the revision theory which entails that the language used is in any way amiss. The revision theorist can say that the reasoning to a contradiction does not proceed in accordance with what is constitutive of the meanings of the expressions of the language. But the meaning-inconsistency theorist's characteristic claim is that the reasoning does so proceed.

I can explain the point in terms of semantic obligations. It is a defining feature of the meaning-inconsistency view that fully competent speakers of natural language take on semantic obligations that cannot be jointly fulfilled. But it is fully compatible with the revision theory that fully competent speakers can fulfill all their semantic obligations: for their semantic obligations need only include accepting that a sentence $S$ is definitionally equivalent to a sentence predicating truth of $S$; not that these sentences are materially equivalent. (One can presumably combine a revision theory with a meaning-inconsistency view. It may for instance be reasonable to see Herzberger's (I982) view as such a combination. My point is just that the revision theory need not be understood as a meaning-inconsistency view, and isn't best seen as such on its most influential elaboration. Neither need the meaning-inconsistency theorist be a revision theorist. Even if the ideas can be conjoined, they are different.)

\section{THE MEANING INCONSISTENCY VIEW AND DIALETHEISM}

Now compare the meaning-inconsistency view with dialetheism; specifically, the dialetheist claim that the proper response to the liar paradox is to accept that there are true contradictions. There are superficial similarities between what I say and what the dialetheist says. The dialetheist too accepts that there is a sense in which our language is inconsistent: it is inconsistent in that some contradictions come out true in it. (In DI I call this Priest-inconsistency.) But the similarities are only superficial. One can hold that the paradoxes show that our language is meaning-inconsistent without taking them to show that our language is Priest-inconsistent. That would be my view. Or one can hold that the paradoxes show that our language is Priest-inconsistent without taking them to show that our language is meaning-inconsistent. That, I took it in DI, is Priest's view. B\&P confirms that impression.

I have argued for the meaning-inconsistency view in a number of papers 4 In DI, I more or less only summarized it. The aim of DI was to compare

\footnotetext{
${ }^{4}$ See primarily my $2002 \mathrm{a}$.
} 
the meaning-inconsistency view with the Priest-inconsistency view. The main points were two. First, I argued that Priest's argument from the liar paradox to dialetheism does not favor a Priest-inconsistency view over a meaninginconsistency view. Second, I argued that once it is plain what sorts of considerations would argue for a Priest-inconsistency view over a meaninginconsistency view, one should start questioning the significance of the Priestinconsistency, even if Priest-inconsistency does obtain.

In brief, my first, and main, argument was this. Take any standard formulation of the liar reasoning. Let the suspects be the assumptions relied on in this reasoning which could prima facie reasonably be singled out as what we should not have accepted in the first place. Standardly, theorists who seek to solve the liar seek to show one of these suspects to be the real culprit. Priest, by contrast, seeks to defend each of the suspects, and on this basis argues that we should accept the contradictory conclusion of the liar reasoning. What I argue is that the suspects are all mcs or immediate consequences of mcs. In holding this, I disagree with orthodox theorists who hold that at least one of the suspects isn't an mc. But I also have a disagreement with Priest. For if meaning-constitutivity doesn't entail analytic truth, then all the suspects can be mcs while still the liar reasoning isn't sound. For it may be that the law of non-contradiction (LNC) is also an mc, so that no matter whether we accept the liar reasoning as sound or we reject one of the underlying assumptions, we end up rejecting one of the mcs. (One complication, which I mention already in DI, is that the dialetheist can accept the LNC in the sense of holding that all its instances are true. It is just that the dialetheist will add that some instances are also false. In order for dialetheism to involve a violation of the relevant mc, the mc must be not only that all instances of the LNC are true, but that they are only true. There are complications here. But since B\&P do not pause on these complications, I won't either.)

\section{REPLY TO B\&P'S CRITICISMS}

Let me now turn to B\&P's criticisms. As mentioned, their main point is that DI is guilty of an ignoratio (pp. 77-78). In DI, I argue that Priest does not manage to show that the assumptions in the liar paradox which jointly entail a contradiction are analytically true; but, в\&P respond, analyticity is beside the point anyway for all that the argument demands is that the assumptions in question are true.

Of course в\&р are right that mere truth is sufficient. But they miss the important point. The arguments Priest (1987) gives for taking the relevant assumptions to be true are arguments of the sort traditionally used to establish analytic truth - for example about the point of a given concept-and then it is relevant that given the distinction between meaning-constitutivity and analytic truth, they establish only meaning-constitutivity. In fact, I needn't have used the notion of analyticity in stating the argument: I could have said sim- 
ply that the arguments of Priest's that aim to establish truth at best establish meaning-constitutivity.

Having dismissed B\&p's charge that I am guilty of an ignoratio, let me now turn to the other major criticisms.

(i) One charge is that since I defend no particular theory of truth, I am 'out of the game' (p. 79). B\&p's making this charge depends on an unwillingness even to engage with what was one main theme in DI. What I argue concerning the liar paradox is that it arises because of the meaning-inconsistency of our language. To say this is not to subscribe to any particular theory of truth alongside dialetheism, the revision theory, fixed-point theories, etc.: any particular claim about the semantic values of the liar sentence and related sentences. Elsewhere-Eklund (manuscript) - I use the following terminology: what I offer is a philosophy of the liar - a general account of why the liar paradox arisesbut I do not thereby immediately take a stand on which theory of the liarwhich view on the semantic values of the terms and sentences concerned-is right.

One argument of mine-the argument that в\&P say commits an ignoratio-is that Priest's argument from the liar paradox to dialetheism does not decide between his theory of the liar and my philosophy, which is in itself neutral among all the theories in the running. I note in DI that Priest can offer other arguments for why one should indeed take the dialetheist route. He can argue either that there is no mc which militates against dialetheism-so there is no reason to take our language to be meaning-inconsistent-or he can argue that even if our language is meaning-inconsistent, a dialetheic, paraconsistent assignment of semantic values is what comes closest to satisfying the mcs. This much I explicitly acknowledge in DI, and I also outline how such arguments might go. Now, although I do say quite a bit more than в\&P give me credit for-oddly, they seem to overlook section IV-I can understand it if they regard me as too dismissive. If my aim were to conclusively demonstrate that Priest-inconsistency is not an option, then surely I say too little. But a large part of my aim in DI was to argue that whereas Priest wants dialetheism to be a deep truth, something which is inescapable because of basic features of thought, the considerations which would be relevant to show that Priestinconsistency, rather than mere meaning-inconsistency, obtains will have to turn on rather specific features of our language or conceptual scheme. (Compare here, by contrast, the rhetoric of e.g. Priest (1995a): true contradictions are the inevitable result of running up against the limits of thought.55

\footnotetext{
${ }^{5} \mathrm{~B} \& \mathrm{P}$ seem to have misunderstood the structure of DI in various ways. For example, they say, 'In section 5 of the paper, Eklund points out that the distinction (between analyticity and meaning-constitutivity) cannot be maintained against what he calls a response-dependent account of meaning, in which the truth of certain sentences just is constituted by our dispositions to accept them' (p. 75). Section 5 is in deed about response-dependence, but the point of the section is not even nearly what $\mathrm{B} \& \mathrm{P}$ say it is. The points that section 5 is concerned to make are: (i) a little discussed argument for dialetheism (and an argument that seems well as strong
} 
So to make explicit my response to B\&p's charge that I am 'out of the game': They are right that there is a particular debate on the liar-indeed, the most central debate-where I do not explicitly have a stand. I do not have, in the terminology earlier introduced, a positive theory of the liar. But of course that does not mean that I do not take a stand on any important issues: I have a philosophy of the liar. The relation to Priest's dialetheism is that the availability of the philosophy of the liar that I defend problematizes his argument for his favored theory.

(ii) в\&P claim that there is a serious lacuna in my account since I lack an explanation of what renders principles meaning-constitutive. в\&P present a supposed dilemma here. They say:

Call a disposition constitutive of competence an sc-disposition. The crucial question concerns what accounts for these. Is it the case that:

I. a speaker possesses sc-dispositions in virtue of a prior grasp of the facts of meaning?

Or is it the case that:

2. a speaker's possession of sc-dispositions is simply a brute fact of semantic competence? (p. 75)

As в\&P rightly stress, I cannot very well give the first answer. So all hinges on whether the second option is workable. When it comes to the second option, $\mathrm{B} \& \mathrm{P}$ present the following argument:

But if we cannot appeal to an independent grasp of meanings, we have a clear problem: How are sc-dispositions to be distinguished from dispositions that hold in virtue of mere belief. People, after all, are disposed to accept many claims that would seem to have little to do with semantic competence. Consider the disposition to accept that the world is older than I minute. All competent speakers of the language barring some malfunction that hinders competency have that disposition. Why is that not an sc-disposition? There would seem to be no principled reason for claiming that it is not. (p. 76)

I find the dialectic a bit curious. For what $\mathrm{B} \& \mathrm{P}$ here present is an argument against any conceptual-role or inferential-role semantics-not just a version of conceptual-role semantics which aims to leave room for meaninginconsistency - and surely they must recognize that they cannot this simply

as those that have been more widely discussed) proceeds via appeal to response-dependence; but (ii) the proper response to this argument is analogous to the response that I provide to the dialetheist's argument from the liar paradox. (I also think that the distinction between analyticity and meaning-constitutivity would only be in tension with an unnecessarily naive response-dependence account. But let me not get into that here.) 
dispose of that whole tradition. There are many things that a defender of conceptual-role semantics can say; and I do not see that the specific features of my view bars me from taking over any of these responses. For example, a conceptual-role semanticist can, following Christopher Peacocke (1992), say that those inferences which a thinker finds 'primitively compelling' are meaning-constitutive, where for an inference to be primitively compelling for a thinker is, roughly, for the thinker to find it compelling and to regard it as not standing in need, even in principle, of further justification. ${ }^{6}$ This promises to distinguish what is genuinely meaning-constitutive from examples like that в\&P provide. And it does not rule out a meaning-inconsistency view, since the principles that lead to trouble in the case of the liar can all be primitively compelling despite the fact that they lead to absurdity. (To stress, I do not mean to subscribe to Peacocke's view. I use it only as a 'model'.) 7

In an accompanying footnote, B\&P go on to say that 'if there's no principled way to distinguish sc-dispositions, then, since all people have different dispositions to assert, it follows that all people speak different languages' (p. $76 \mathrm{fn} 3$ ). I have already dismissed the antecedent of this claim. But a version of their worry may still remain: if different speakers find different inferences primitively compelling, different speakers will speak different languages. But the conceptual role theorist has the tools to deal with this. She can appeal to a distinction between expressions we are fully competent with and those expressions which we use with their customary meanings only because of how we defer to other speakers. I did not go into these problems in the relevant papers of mine because the problems arise equally for all theories which are, broadly speaking, conceptual-role theories. There are further peculiarities in the relevant part of в\&P's discussion. With no textual support whatever, they ascribe to me a behaviorist view. And they present a compositionality worry which, whether serious or not, arises equally for all versions of conceptual-role semantics.

There are, to state the obvious, objections one may have to the specific view I hold. And there are also important criticisms of conceptual role theories generally. It would be silly not to take such criticisms seriously. All I want to stress here is that the relevant criticisms in B\&P are criticisms that, insofar as they are worth taking seriously at all, are criticisms relevant to all conceptualrole theories; and moreover that there are known responses to these criticisms in the literature. It is not obvious that the responses work. But B\&P do nothing to advance the case against conceptual-role theories.

\footnotetext{
${ }^{6}$ See Peacocke (I992).

${ }^{7}$ For further discussion of the meaning-inconsistency view and semantic competence, see my (2007).
} 


\section{THE STRENGTHENED LIAR}

B\&P point to the strengthened liar as providing a reason to favor a dialetheist theory over a non-dialetheist theory (p. 79). This is indeed a matter it would have been nice to have explicitly discussed in DI, both because Priest has often pointed to the strengthened liar as providing an argument for his dialetheist view and I should have countered this specific argument, and, more importantly, because attention to the strengthened liar positively helps my case. The meaning-inconsistency view helps diagnose what strengthened liar problems are all about.

A strengthened liar problem (or revenge problem) for a given solution to the liar paradox is, roughly, a liar-like problem that arises for the solution when we focus on a new liar-like sentence formulated using the apparatus of the purported solution. One common response to these problems is to insist that properties expressed by the technical terms used in stating the given solution can be expressed only in a richer metalanguage.

What Priest argues is that all non-dialetheist solutions face strengthened liar problems, but a dialetheist account can avoid these problems and accordingly is preferable. There are a number of ways for a non-dialetheist to attempt to respond to Priest's argument. One is to insist that a given non-dialetheist account does not in fact face a strengthened liar problem. A second is to insist that the dialetheist too faces problems as serious as the strengthened liar problem. For example, what can the dialetheist say about a liar sentence that says of itself that it is only untrue? (See e.g. Terence Parsons (I990) and Priest (I995a) for discussion.) Thirdly, one can problematize the issue of exactly what is the problem with strengthened liars and with being committed to saying that certain properties aren't expressible in our actual language. (For further discussion of these issues, see further Eklund (2008).) It is the third line of argument I want to focus on here. Consider a theory of truth-call it T-which is committed to saying that a given property $\mathrm{P}$ isn't expressible in our actual language. Why exactly would T's saying this amount to an embarrassment? Here are some possible claims that may be made.

(a) Natural language is universal. So all properties are expressible in our natural language. Hence, $T$ must be false.

(b) $\mathrm{T}$ is itself formulated in our actual language and the formulation of $\mathrm{T}$ involves an expression which expresses $\mathrm{P}$. Then $\mathrm{T}$ is self-defeating.

(c) Property $\mathrm{P}$ is obviously expressible in natural language, so any theory which denies that it is falls on its own absurdity.

I think we have little reason to believe that natural language is universal, so if a theory like $T$ faces problems, that must be because of (b) or (c) (Again see further my (2008).) 
With this as background, let me turn to the consequences of the meaninginconsistency view for how to think about strengthened liar problems and appeal to inexpressibility. The meaning-inconsistency view affords a nice explanation of how strengthened liar problems arise, and make such problems in some ways less serious. Here is the basic idea.

If the mcs for the expressions of a given language cannot be jointly satisfied, then the semantic values of some expressions will be such that the mcs for these expressions are not true. Say that the relevant expressions misfire. (Intuitively: the semantic values of these expressions aren't what we intend them to be.) It is then a consequence of my view on the liar that some expressions centrally employed in the liar paradox will misfire. But if we keep in mind that the liar paradox has the consequence that some expressions misfire, the strengthened liar problem is to be expected: any account which does relatively well in assigning intuitively acceptable semantic values to some of the expressions centrally employed in the liar reasoning will assign counterintuitive semantic values to other expressions. That is what is shown by strengthened liar problems. (The dialetheist has the seeming advantage here that she can allow an inconsistent assignment of semantic values. But not even the dialetheist can respect all mcs, for she cannot respect what is meaning-constitutive for negation. This is the main point from DI again.)

Compare perhaps vagueness, and the problem of higher-order vagueness. I hold a view on vagueness and the sorites paradox like that I hold on the liar paradox. Focus on vague predicates. I think that for $\mathrm{F}$ a vague predicate, a principle of the following form-a tolerance principle-is meaning-constitutive for F:

A small enough difference in F's parameter of application never matters to the applicability of $F$, while there is a difference in the parameter of application that sometimes matters 8

As an example, consider 'tall'. According to a plausible tolerance principle for 'tall', a difference in height (the parameter of application in this case) of one angstrom never matters while a difference in height of three inches sometimes matters.

Consider now what is known as the problem of higher-order vagueness. Take a sorites series for a vague predicate $F$ : a series of objects $1, \ldots, n$ such that for each pair of adjacent objects, the objects in this pair differ only very slightly in F's parameter of application and such that $F(1)$ is, intuitively, obviously true and $F(n)$ is, intuitively, obviously false. It is then counterintuitive that there should be some objects $i$ and $i+1$ such that $F(i)$ is true and $F(i+1)$ is false. In response many theorists opt for deviant logic and semantics. Some theorists of vagueness say that when object $i$ is borderline $F, F(i)$ is neither true nor

\footnotetext{
${ }^{8}$ See my (2002a) and (2005) for more on vagueness. The notion of tolerance is from Wright (1975).
} 
false. Other theorists of vagueness posit a continuum of truth-values between 1 (True) and 0 (False). Both types of accounts face a problem of higher-order vagueness. Saying that $F$ divides up objects $1, \ldots, n$ into three classes-true, false and in between-is really hardly more plausible than saying that $F$ divides them up into two classes (true and false). 9

Now, the fact that the problem of higher-order vagueness is nicely explained by the view that vagueness is a matter of meaning-inconsistency. For it is a consequence of the view that vagueness is a matter of meaninginconsistency that all assignments of semantic values to vague expressions will be counterintuitive-for no assignment of semantic values makes true the tolerance principles which are mcs for vague expressions. There will always be a bump in the carpet somewhere.

Suppose now that the diagnosis of strengthened liar problems afforded by the meaning-inconsistency view is correct. Then strengthened liar problems should in general seem less worrisome, and the dialetheist's argument from the strengthened liar should look less impressive. Go back to the list of alternatives (a)-(c) above. I have already dismissed (a). Given the diagnosis of the strengthened liar, we can say the following about (b) and (c). If expressions can misfire in the way indicated, there are two questions one may ask about a given property and given language L. (I) Is there in L an expression that actually expresses the property in question? (2) Is there in $\mathrm{L}$ an expression that, so to speak, aims to express the property, in the sense that if it does not express the property that is because it misfires? Let us say that if the answer to the first question is affirmative, the property is expressible in $\mathrm{L}$, and if the answer to the second question is affirmative, the property is quasi-expressible in L. Given the notion of quasi-expressibility, a theorist defending theory $T$ can insist that the relevant property still is quasi-expressible. She can say, in response to a problem of the form (c), 'I agree that it seems perfectly obvious that $\mathrm{P}$ is expressible in our actual language. But I can account for this as follows. We have an expression of our language which aims to express $\mathrm{P}$-an expression of our language which misfires if it doesn't express P. On my view, however, this expression misfires.' And she can say in response to a problem of the form (b), 'All that the strengthened liar shows is that one of the expressions that I use in stating my solution misfires. Maybe the real lesson of the strengthened liar is that misfiring is something we just have to learn to accept as a fact.' (Of course, some expressions used in these speeches will themselves misfire. But that is part of the package.)

Needless to say, there is more to say about this response to strengthened liar problems. What I want to emphasize here is just that the view on the liar paradox that I am defending affords a new and promising way to look at

\footnotetext{
${ }^{9}$ There are of course responses to the problem of higher-order vagueness. One is to urge the necessity of a vague metalanguage. Since I am bringing up vagueness only as an illustration, I will not here discuss those responses.
} 
strengthened liar problems.

\section{REFERENCES}

[i] beall, JC and graham priest (2007), 'Not So Deep Inconsistency: A Reply to Eklund', Australasian fournal of Logic 5: 74-84, http://www . philosophy.unimelb.edu.au/ajl/2007/.

[2] eklund, matti (2002a), 'Deep Inconsistency', Australasian fournal of Philosophy 80: 32I-3I.

[3] eklund, Matti (2002b), 'Inconsistent Languages', Philosophy and Phenomenological Research 64: 25I-75.

[4] eklund, matti (2005), 'What Vagueness Consists In', Philosophical Studies $125: 27-60$.

[5] EKLUND, MatTi (2007), 'Meaning-Constitutivity', Inquiry 50: 559-74.

[6] eklund, matti (2008), 'The Liar Paradox, Expressibility, Possible Languages', in JC BEALL (ed), The Revenge of the Liar, Oxford: Oxford University Press.

[7] EKLUND, MATTI (manuscript), 'Metasolutions to the Liar'.

[8] gupta, anil and nuel belnap (1993), The Revision Theory of Truth, Cambridge, Massachusetts: Mit Press.

[9] herzberger, hans (1982), 'Naive Semantics and the Liar Paradox', fournal of Philosophy 79: 479-97.

[io] parsons, terence (I990), 'True Contradictions', Canadian fournal of Philosophy 20: 335-53.

[ii] peacocke, Christopher (I992), A Study of Concepts, Cambridge, Massachusetts: Mit Press.

[i2] priest, graham (1987), In Contradiction, Dordrecht: Martinus Nijhoff Publishers. (2nd edition published 2006 by Oxford University Press.)

[13] Priest, Graham (1995a), Beyond the Limits of Thought, Cambridge: Cambridge University Press.

[i4] Priest, graham (i995b), 'Gaps, and Gluts: Reply to Parsons', Canadian Fournal of Philosophy 25: 57-66.

[15] tappenden, Jamie (I993), 'The Liar and Sorites Paradoxes: Toward a Unified Treatment', fournal of Philosophy 90: 55 $1-577$. 
[i6] wright, Crispin (1975) 'On the Coherence of Vague Predicates', Synthese 30: $325-65$. 
The Australasian fournal of Logic (ISSN I448-5052) disseminates articles that significantly advance the study of logic, in its mathematical, philosophical or computational guises. The scope of the journal includes all areas of logic, both pure and applied to topics in philosophy, mathematics, computation, linguistics and the other sciences.

Articles appearing in the journal have been carefully and critically refereed under the responsibility of members of the Editorial Board. Only papers judged to be both significant and excellent are accepted for publication.

The journal is freely available at the journal website at

$$
\text { http://www.philosophy.unimelb.edu.au/ajl/ }
$$

All issues of the journal are archived electronically at the journal website.

SuBSCRIPTIONS Individuals may subscribe to the journal by sending an email, including a full name, an institutional affiliation and an email address to the managing editor at ajl-editors@unimelb.edu.au Subscribers will receive email abstracts of accepted papers to an address of their choice. For institutional subscription, please email the managing editor at ajl-editors@unimelb.edu.au

Complete published papers may be downloaded at the journal's website at http: //www.philosophy.unimelb.edu.au/ajl/ The journal currently publishes in pdf format.

Submission The journal accepts submissions of papers electronically. To submit an article for publication, send the $\mathrm{LT}_{\mathrm{E}} \mathrm{X}$ source of a submission to a member of the editorial board. For a current list of the editorial board, consult the website.

The copyright of each article remains with the author or authors of that article. 